\title{
Multigrid Convergent Curvature Estimator
}

\author{
Christophe Fiorio ${ }^{1}$, Christian Mercat $^{3}$, and Frédéric Rieux ${ }^{1,2}$ \\ 1 LIRMM, Université Montpellier 2, \\ F-34392 MONTPELLIER, France \\ 2 I3M, Université de Montpellier 2 c.c. 51 \\ F-34095 Montpellier Cedex 5, France \\ 3 S2HEP EA 4148 Université Claude Bernard Lyon 1 \\ F-69622 Villeurbanne cedex, France
}

\begin{abstract}
We propose in this paper an estimator of derivative and curvature of discrete curves. Based on adaptive convolution that preserves contour, we use local geometrical information as the heat kernel to convolve with a discrete curve and give estimation of its geometrical parameters. We recover on regular part of the curve the classical convolution based on gaussian kernel. We study the bounded error of our approach for first and second order derivative and we discuss about the multigrid convergence.
\end{abstract}

\section{Introduction}

Curvature is a geometrical invariant of shapes or curves which characterizes the object. It has a clear definition in the smooth setting but on discrete shapes, it is an important problem to give an estimation of this invariant and there exists many approaches.

A geometrical definition, based on digital segment decomposition of the shape, estimating the first derivative of the curve is given in [610. Digital circle arcs decomposition is used in 31615 . Decomposition into maximals segments has been proved multigrid convergent, meaning that when the digital discretization step tends to zero, the derivative estimation converges toward the underlying continuous one. This approach has been generalized by using maximal digital circle arcs in [15]. The authors studied the multigrid convergence according to the length of the digital circle arcs when the discretization step tends to zero, and conjectured the multigrid convergence of their approach. Segmentation with nonprimitive objects has been studied in [14] where the authors proved the multigrid convergence. Others approaches have been proposed, based on convolution by a Gaussian kernel in [129] or a kernel adapted to the contour in [7]. Gaussian kernel has been widely studied in Image analysis to reduce noise effect [17]. Although Gaussian kernel is optimal on flatten parts (Theorem of Gabor [11]), its has a blurring on the contour. For this reason a large amount of works deals with adaptive kernels to reduce the blurring effect whereas still reducing the intensity of the noise. We have proposed in a previous work a digital and adaptive kernel suited for curves and surfaces [7/8], but we were not able to prove the multigrid

R. Gonzalez-Diaz, M.-J. Jimenez, B. Medrano (Eds.): DGCI 2013, LNCS 7749, pp. 395-406, 2013.

(C) Springer-Verlag Berlin Heidelberg 2013 
convergence of this approach. On the other hand, the gaussian kernel has been proved multigrid convergent for $\mathcal{C}^{3}$ curves in [12] and for $\mathcal{C}^{2}$ curves in [5] for derivative of order one.

We propose in this paper to study the multigrid convergence of derivatives of order one and two on curves. We rely on convolution estimators (Section 2) with Least square methods to give an estimation of the derivative (Section 3). With the help of a previous work [2], we will use the link between the two approaches to show that estimation based on adaptive kernel is multigrid convergent (Section 4), and finally we will propose numerical examples of curvature estimation on digital curves (Section 51).

\section{Convolution Derivatives Estimator}

\subsection{Convolution with Gaussian Kernel}

Definition 1. Convolution Product

Let $F: \mathbb{Z} \longrightarrow \mathbb{Z}$ and $K: \mathbb{Z} \longrightarrow \mathbb{Z}$ be two discrete functions. We call the convolution product of $F$ by $K$ denoted $F * K$ the function:

$$
\begin{aligned}
F * K: \mathbb{Z} & \rightarrow \mathbb{Z} \\
a & \mapsto \sum_{i \in \mathbb{Z}} F(a-i) K(i)
\end{aligned}
$$

Definition 2. Derivative Estimation[12]

Let $\varphi$ be a discrete function. An estimation of the first derivative of $\varphi$ at point $x$ is given by:

$$
\left(\Delta_{2 m-1} * \varphi\right)(x)=\frac{1}{2^{2 m-1}} \sum_{i=-m+1}^{m}\left(\begin{array}{c}
2 m-1 \\
m-1+i
\end{array}\right)(\varphi(n+i+1)-\varphi(n-1+i) \gamma(3)
$$

Theorem 3. [5]

Let $f: \mathbb{R} \rightarrow \mathbb{R}$ be a $C^{3}$ bounded function, let $\left.\left.\alpha \in\right] 0,1\right], K \in \mathbb{R}_{+}^{*}$ and $h \in \mathbb{R}_{+}^{*}$. We suppose that $\Gamma: \mathbb{Z} \rightarrow \mathbb{Z}$ is such that $|h \Gamma(i)-f(h i)| \leq K h^{\alpha}$. Then for $m=h^{2(\alpha-3) / 3}$ we have $\left|\left(D_{2 m-1} * u\right)(n)-f^{\prime}(n h)\right| \in O\left(h^{2 \alpha / 3}\right)$

We have proposed in [7/8 to study an adaptive kernel for derivative estimation. Gaussian Kernel previously introduced has been widely used in image analysis but its blurring effect destroy fine structures and boundaries. Nevertheless, Gabor [11] shows that on flatten part of an image gaussian kernel are the most efficient masks to reduce noise. Many works deal with an adaptive kernels that preserve contours while reducing noise [131]. Along these lines, we propose this discrete kernel which is gaussian on regular parts of a curve and adaptive on high curvature points. 


\subsection{Convolution with Adaptive Kernel}

We recall the definition of our adaptive kernel. It is a weighted version of the classical adjacency matrix.

Definition 4. Adaptive Kernel

Let $\Sigma \subset \mathbb{Z}^{n}$ be a sets of 0 -connected voxels. We define on $\Sigma$ the Markov chain in discrete time whose states are $\mathcal{E}$, the voxels of $\Sigma$, and whose transitions between two neighbors are constrained by:

- Probability $\frac{1}{2^{n}}$ to go from center of the voxel to one of its corner

- Equiprobable repartitions from the corner to every adjacent voxel.

We call $A_{s}$ the adjacency matrix of the adaptive digital diffusion process. Many properties of this digital diffusion process can be found in 788 .

Definition 5. First derivative estimation

Let $\varphi$ be a discrete function and $A_{s}^{m}$ the adaptive kernel computed on $\varphi$. Its first derivative estimation at point $n$ is given by:

$$
\left(D_{(2 m+1)}^{1} * \varphi\right)(n)=\sum_{i=-m}^{m} A_{s}^{m}(n, i)(\varphi(n+i+1)-\varphi(n-1+i))
$$

Following Theorem. 3 we will show that the estimator error is bounded an converges toward zero when the discretization step tends to zero. We use the least square method to tackle the problem.

\section{Least Square Approximation}

\subsection{Definitions}

Let $\left\{y_{0}, y_{1}, \ldots, y_{n}\right\}$ and $\left\{x_{0}, x_{1}, \ldots, x_{n}\right\}$ be two matching sets of experimental measures. We search a relation between $x_{k}$ and $y_{k}$ for $k \in[1, n]$. In the case of derivative estimation, we are looking for the line fitting the set and passing through a point $\left(x_{i}, y_{i}\right) i \in[1, n]$. Classical least square approximation is to compute the minimum of the following sum $S(a)$ with respect to $a$, the slope of the target line, with

$$
S(a)=\sum_{j=1}^{n}\left(\left(y_{j}-y_{i}\right)-a\left(x_{j}-x_{i}\right)\right)^{2}
$$

In our situation, the diffusion process defines a reliability weight between two given points: Let $A_{s}^{m}(i, j)$ the weight at point $j$ starting from point $i$ :

$$
S(a)=\sum_{j=1}^{n}\left(\left(y_{j}-y_{i}\right)-a\left(x_{j}-x_{i}\right)\right)^{2} A_{s}^{m}(i, j)
$$


Then the optimal slope $a$ is the solution of

$$
\begin{aligned}
\frac{\partial S}{\partial a} & =\sum_{j=1}^{n} 2\left(\left(y_{j}-y_{i}\right)-a\left(x_{j}-x_{i}\right)\right) \times\left(x_{j}-x_{i}\right) A_{s}^{m}(i, j)=0 \\
& =\sum_{j=1}^{n}\left(y_{j}-y_{i}\right)\left(x_{j}-x_{i}\right) A_{s}^{m}(i, j)-a \sum_{j=1}^{n}\left(x_{j}-x_{i}\right)^{2} A_{s}^{m}(i, j)=0 \\
a & =\frac{\sum_{j=1}^{n}\left(y_{j}-y_{i}\right)\left(x_{j}-x_{i}\right) A_{s}^{m}(i, j)}{\sum_{j=1}^{n}\left(x_{j}-x_{i}\right)^{2} A_{s}^{m}(i, j)}
\end{aligned}
$$

Definition 6. Let $\mathcal{C}=\left\{c_{0}, c_{1}, \ldots, c_{n}\right\}$ be a discrete curve and $A_{s}^{m}$ the adaptive kernel associated with $\mathcal{C}$. We call the least square estimation in $c_{i}=\left(x_{i}, y_{i}\right)$, the line of slope a such that:

$$
\begin{aligned}
& a=\frac{\sum_{j=1}^{n}\left(y_{j}-y_{i}\right)\left(x_{j}-x_{i}\right) A_{s}^{m}(i, j)}{\sum_{j=1}^{n}\left(x_{j}-x_{i}\right)^{2} A_{s}^{m}(i, j)} \\
& b=y_{i}-a x_{i}
\end{aligned}
$$

In the next section, we will study the link between the least square approach and the one based on the associated weighted convolution.

\subsection{Link with Gaussian Convolution}

The classical least square derivative approach is proved to be multigrid convergent for $\mathcal{C}^{2}$ curves [2]. The authors also generalize this results to second order derivative functions. We will add geometrical informations of weights in the least square approach and we will prove that the formula is related to the gaussian convolution.

Theorem 7. Let $\mathcal{C}=\left\{c_{0}, c_{1}, \ldots, c_{n}\right\}$ be a discrete curve such that $c_{j}=\left(x_{j}, y_{j}\right)$ and $A_{s}$ the stochastic matrix associated with $\mathcal{C}$. If $A_{s}^{m}(i, j)=\frac{1}{2^{2 m-1}} C_{2 m+1}^{m-j+1}$, then we have:

$$
\frac{\sum_{j=0}^{2 m}\left(y_{j}-y_{i}\right)\left(x_{j}-x_{i}\right) A_{s}^{m}(i, j)}{\sum_{j=0}^{2 m}\left(x_{j}-x_{i}\right)^{2} A_{s}^{m}(i, j)}=D_{2 m-1}^{1}\left(c_{i}\right)
$$

Remark 8. This theorem allows us to connect the gaussian weighted least square approach and the gaussian convolution. Note that the theorem only holds for gaussian weights but we will see how to deal with adaptive weights. 
Proof. We rewrite the least square estimation formula to simplify the proof. Without loss of generality, we suppose that the estimation tangent point is centered at 0 . Let $p_{j}=A_{s}^{m}(i, j)$

$$
a=\frac{\sum_{j=-m}^{m}\left(y_{j}-y_{0}\right) j p_{j}}{\sum_{j=-m}^{m} j^{2} p_{j}}
$$

If $p_{j}$ is given by binomial numbers, we have:

$$
a=\frac{\sum_{j=-m}^{m}\left(y_{j}-y_{0}\right) j C_{2 m-1}^{m+j}}{\sum_{j=-m}^{m} j^{2} C_{2 m}^{m+j}}
$$

We easily check that $\sum_{j=-m}^{m} j^{2} C_{2 m}^{m+j}=m 2^{2 m-1}$.

We collect $y_{j}$ in:

$$
\begin{aligned}
\left(D_{(2 m+1)}^{1} * \varphi\right)(x) & =\sum_{i=0}^{2 m+1} C_{2 m-1}^{m+j}\left(y_{j+1}-y_{j-1}\right) \\
C_{2 m-1}^{m+j}\left(-y_{j}\right)+C_{2 m-1}^{m+j-1}\left(y_{j}\right) & =y_{j}\left(\frac{(2 m-1) !}{(m+j-1) !(m+j) !}-\frac{(2 m-1) !}{(m+j) !(m-j-1) !}\right) \\
& =y_{j} \frac{(2 m) !}{2 m} \frac{m+j-(m-j)}{(m+j) !(m-j) !} \\
& =y_{j} \frac{1}{2 m} \frac{2 j(2 m) !}{(m+j) !(m-j) !} \\
& =y_{j} \frac{j}{m} C_{2 m}^{m+j}
\end{aligned}
$$

We will use this result to show the link with our adaptive kernel.

\subsection{Link with Adaptive Kernel}

First we need technical results about our adaptive kernel.

Theorem 9. [7] Let $\mathcal{D}(a, b, \mu, \omega)$ be a discrete line and let $A_{s}$ be its adaptive kernel. Then $\frac{1}{\sqrt{2 m+1}} A_{s}^{m}(0) \underset{m \rightarrow \infty}{\stackrel{\mathcal{L}}{\longrightarrow}} \mathcal{N}(0,1)$.

This theorem shows that on discrete lines, the adaptive kernel follows a normal law; the adaptive kernel has, in the limit, the same statistic distribution as the gaussian kernel.

Proposition 10. The discrete standard normal diffusion, on the sampling of a curve of continuous curvature, converges in law toward the centered normalized normal law. 
More precisely:

Lemma 11. Let $f \in \mathcal{C}^{2}$, Let $x \in \mathcal{D}_{f}$ and $\sigma_{x}$ be the standard deviation of diffusion on a discrete line of slope $f^{\prime}(x)$. Let $i_{\epsilon}$ be the discrete point associated to $x$ on $\varphi_{\epsilon}: \mathbb{Z} \longrightarrow \mathbb{Z}$ discretization of $f$ with a step $\epsilon, A_{s}$ the convolution kernel associated to $\varphi_{\epsilon}$ and $p_{\epsilon}$ the length of the maximal segment centered at $i_{\epsilon}$. We choose $m_{\epsilon}$ such that $\frac{p_{\epsilon}}{2}>m_{\epsilon}>\frac{p_{\epsilon}}{4}$ and we note $X_{\epsilon}(y)=\frac{1}{m \sigma_{x}} A_{s}^{m}\left(i_{\epsilon}, i_{\epsilon}+\left\lfloor\frac{y m \sigma_{x}}{\epsilon}\right\rfloor\right)$, the standard normal diffusion process at $i_{\epsilon}$. Then $\left(X_{\epsilon}\right)_{\epsilon \in \mathbb{R}}$ converges in law toward the standard normal law:

$$
X_{\epsilon} \underset{\epsilon \rightarrow 0}{\stackrel{\mathcal{L}}{\longrightarrow}} \mathcal{N}(0,1)
$$

Remark 12. This proposition refers to the asymptotic distribution of the diffusion process for large time being equivalent to a normal law for a given standard deviation. Therefore, on a curve of continuous curvature, the adaptive kernel converges toward the gaussian kernel.

Proof. The starting point of diffusion $i_{\epsilon}$ belong to $S$, maximal segment centered at $i_{\epsilon}$ with length $p_{\epsilon}$. Statistical distribution of weights starting from point $i_{\epsilon}$ have a standard deviation $\sigma_{\epsilon}$. Because of the class of the function $f \in \mathcal{C}^{2}$, the deviation $\sigma_{\epsilon}$ actually converges, when $\epsilon \rightarrow 0$, toward a number $\sigma_{x}$, which is the standard deviation of the discrete diffusion on the discrete line of slope $f^{\prime}(x)$.

For $j$ such that $\left|i_{\epsilon}-j\right|<p_{\epsilon}$, being on a maximal segment, the standard deviation of the process between $i_{\epsilon}$ and $j$ for a time $m<p_{\epsilon}$ is identical to the process on the discrete line $S$.

According to theorem. 9] the standard normal diffusion process on the discrete line $S$ converges in law toward the normal law when $m \rightarrow \infty$. And according to [10], $p_{\epsilon}$ is not bounded when $\epsilon \rightarrow 0$. Then for $\frac{p_{\epsilon}}{2}>m_{\epsilon}>\frac{p_{\epsilon}}{4}$,

$$
X_{\epsilon} \underset{\epsilon \rightarrow 0}{\stackrel{\mathcal{L}}{\longrightarrow}} \mathcal{N}(0,1)
$$

\section{Multigrid Convergence of Derivatives Estimator}

In this section, we study the asymptotic convergence of the derivative estimator of order one and two. In the last section, we study the link between convolution and least square approach. We will use this link to prove the multigrid convergence of our approach. In [2], the authors propose a proof of multigrid convergence of least square approach without ponderation. We extend their work to the gaussian approach and our adaptive approach. The need for this extension has been already documented in image analysis: the constant averaging mask is not efficient, the gaussian mask taking into account the distance from the treated pixel is better, and to preserve contours, one needs an adaptive mask that takes into account the geometry of the image. We propose a similar approach on discrete curves, and in a forthcoming paper we will apply it to gray level images. 


\subsection{Theorical Convergence}

Theorem 13. Let $y_{i}=D^{1}(f)\left(x_{i}\right), x_{i} \in\left\{x_{1}, x_{2}, \ldots, x_{n}\right\}$ be the discretisation of a functiony $=f(x) \mathcal{C}^{2}$. Then $\forall j, \exists \zeta_{j} \in\left[x, x_{j}\right]$ such that:

$$
\forall k, \quad\left|D^{1}(f)\left(x_{k}\right)-f^{\prime}\left(x_{k}\right)\right| \leq \max _{x_{j} \in \mathcal{V}_{k}}\left\{\left|\frac{f^{\prime \prime}\left(\zeta_{j}\right)}{2}\left(x_{j}-x_{k}\right)\right|\right\}
$$

Proof. Under the hypothesis that $f$ is $\mathcal{C}^{2}$, we have a Taylor expansion in $x_{k}$ : $\forall x \in \mathbb{R}, \exists \zeta \in\left[x, x_{k}\right]$

$$
f(x)=f\left(x_{k}\right)+f^{\prime}\left(x_{k}\right)\left(x-x_{k}\right)+\frac{f^{\prime \prime}(\zeta)}{2}\left(x-x_{k}\right)^{2}
$$

$\forall j, \exists \zeta_{j}$ with a weight $p_{j}$ such that:

$$
y_{j}-y_{k}=f\left(x_{j}\right)-f\left(x_{k}\right)=f^{\prime}\left(x_{k}\right)\left(x_{j}-x_{k}\right)+\frac{f^{\prime \prime}\left(\zeta_{j}\right)}{2}\left(x_{j}-x_{k}\right)^{2}
$$

Bringing in Equation 8 into the definition of the derivative, we get:

$$
\begin{aligned}
D^{1}(f)\left(x_{k}\right) & =\frac{\sum_{j=1}^{n}\left(y_{j}-y_{k}\right)\left(x_{j}-x_{k}\right) p_{j}}{\sum_{j=1}^{n}\left(x_{j}-x_{k}\right)^{2} p_{j}} \\
& =\frac{\sum_{j=1}^{n} f^{\prime}\left(x_{k}\right)\left(x_{j}-x_{k}\right)^{2} p_{j}+\frac{f^{\prime \prime}\left(\zeta_{j}\right)}{2}\left(x_{j}-x_{k}\right)^{3} p_{j}}{\sum_{j=1}^{n}\left(x_{j}-x_{k}\right)^{2} p_{j}} \\
& =f^{\prime}\left(x_{k}\right)+\frac{\sum_{j=1}^{n} \frac{f^{\prime \prime}\left(\zeta_{j}\right)}{2}\left(x_{j}-x_{k}\right)^{3} p_{j}}{\sum_{j=1}^{n}\left(x_{j}-x_{k}\right)^{2} p_{j}} .
\end{aligned}
$$

Then,

$$
\begin{aligned}
\left|D^{1}(f)\left(x_{k}\right)-f^{\prime}\left(x_{k}\right)\right| & =\left|\frac{\sum_{j=1}^{n} \frac{f^{\prime \prime}\left(\zeta_{j}\right)}{2}\left(x_{j}-x_{k}\right)^{3} p_{j}}{\sum_{j=1}^{n}\left(x_{j}-x_{k}\right)^{2} p_{j}}\right| \\
& \leq \frac{\sum_{j=1}^{n} \max _{j \in \mathcal{V}_{k}}\left\{\left|\frac{f^{\prime \prime}\left(\zeta_{j}\right)}{2}\left(x_{j}-x_{k}\right)\right|\right\}\left(x_{j}-x_{k}\right)^{2} p_{j}}{\sum_{j=1}^{n}\left(x_{j}-x_{k}\right)^{2} p_{j}} \\
& \leq \max _{j \in \mathcal{V}_{k}}\left\{\left|\frac{f^{\prime \prime}\left(\zeta_{j}\right)}{2}\left(x_{j}-x_{k}\right)\right|\right\} .
\end{aligned}
$$

With a similar approach, we have a bounded error for derivatives of order two:

Theorem 14. Let $y_{i}=D^{2}(f)\left(x_{i}\right), x_{i} \in\left\{x_{1}, x_{2}, \ldots, x_{n}\right\}$ be the discretization of a function $y=f(x)$ defined on $I \mathcal{C}^{3}$. We note $h$ the discretization step and $\Delta_{i k}=\frac{\left(x_{i}-x_{k}\right)^{3} p_{i}}{\sum_{i=1}^{n}\left(x_{i}-x_{k}\right)^{2} p_{i}}$. Then $\forall i, j, k, \exists \zeta_{j} \in\left[x, x_{j}\right], \exists \epsilon_{i} \in\left[x, x_{i}\right]$ et $\exists \epsilon_{i}^{\prime} \in\left[x, x_{i}^{\prime}\right]$ such that:

$$
\left|D^{2}(f)\left(x_{k}\right)-f^{\prime \prime}\left(x_{k}\right)\right| \leq \max _{j \in \mathcal{V}_{k}}\left\{\left|\frac{f^{\prime \prime \prime}\left(\zeta_{j}\right)}{2}\left(x_{j}-x_{k}\right)\right|\right\}+\max _{i, j \in \mathcal{V}_{k}}\left\{\frac{n}{\left(x_{j}-x_{k}\right) h} f^{\prime \prime}\left(\epsilon_{i}\right) \Delta_{i k}-f^{\prime \prime}\left(\epsilon_{i}^{\prime}\right) \Delta_{i j}\right\}
$$


Proof. Let $x_{k} \in I$, the second derivative at point $k$ is given by:

$$
D^{2}(f)\left(x_{k}\right)=\frac{\sum_{j=1}^{n}\left(D^{1}(f)\left(x_{j}\right)-D^{1}(f)\left(x_{k}\right)\right)\left(x_{j}-x_{k}\right) p_{j}}{\sum_{j=1}^{n}\left(x_{j}-x_{k}\right)^{2} p_{j}}
$$

Identically to the last proof, $\exists \zeta_{j} \in\left[x, x_{j}\right]$ such that:

$$
f^{\prime}\left(x_{j}\right)-f^{\prime}\left(x_{k}\right)=f^{\prime \prime}\left(x_{k}\right)\left(x_{j}-x_{k}\right)+\frac{f^{\prime \prime \prime}\left(\zeta_{j}\right)}{2 !}\left(x_{k}-x_{j}\right)^{2}
$$

Identically to Theorem 13 , we have:

$$
\begin{gathered}
\exists \zeta_{i} \in\left[x, x_{i}\right], \quad D^{1}(f)\left(x_{k}\right)=f^{\prime}\left(x_{k}\right)+\frac{\sum_{i=1}^{n} \frac{f^{\prime \prime}\left(\zeta_{i}\right)}{2}\left(x_{i}-x_{k}\right)^{3} p_{i}}{\sum_{i=1}^{n}\left(x_{i}-x_{k}\right)^{2} p_{i}} \\
\exists \zeta_{i}^{\prime} \in\left[x, x_{i}^{\prime}\right], \quad D^{1}(f)\left(x_{j}\right)=f^{\prime}\left(x_{j}\right)+\frac{\sum_{i=1}^{n} \frac{f^{\prime \prime}\left(\zeta_{i}^{\prime}\right)}{2}\left(x_{i}-x_{j}\right)^{3} p_{i}}{\sum_{i=1}^{n}\left(x_{i}-x_{j}\right)^{2} p_{i}} \\
f^{\prime}\left(x_{j}\right)-f^{\prime}\left(x_{k}\right)=f^{\prime \prime}\left(x_{k}\right)\left(x_{j}-x_{k}\right)+\frac{f^{\prime \prime \prime}\left(\zeta_{j}\right)}{2}\left(x_{j}-x_{k}\right)^{2} \\
\left(f^{\prime}\left(x_{j}\right)-f^{\prime}\left(x_{k}\right)\right)\left(x_{j}-x_{k}\right)=f^{\prime \prime}\left(x_{k}\right)\left(x_{j}-x_{k}\right)^{2}+\frac{f^{\prime \prime \prime}\left(\zeta_{j}\right)}{2}\left(x_{j}-x_{k}\right)^{3} \\
\left(D^{1}(f)\left(x_{j}\right)-D^{1}(f)\left(x_{k}\right)\right)\left(x_{j}-x_{k}\right)=f^{\prime \prime}\left(x_{k}\right)\left(x_{j}-x_{k}\right)^{2}+\frac{f^{\prime \prime \prime}\left(\zeta_{j}\right)}{2}\left(x_{j}-x_{k}\right)^{3}+\left(x_{j}-x_{k}\right) \\
\left\{\frac{\sum_{i=1}^{n} \frac{f^{\prime \prime}\left(\zeta_{i}\right)}{2}\left(x_{i}-x_{k}\right)^{3} p_{i}}{\sum_{i=1}^{n}\left(x_{i}-x_{k}\right)^{2} p_{i}}-\frac{\sum_{i=1}^{n} \frac{f^{\prime \prime}\left(\zeta_{j}^{\prime}\right)}{2}\left(x_{i}-x_{j}\right)^{3} p_{i}}{\sum_{r=1}^{n}\left(x_{i}-x_{j}\right)^{2} p_{i}}\right\}\left(x_{j}-x_{k}\right)
\end{gathered}
$$

Bringing Equation 12 into Equation 9,

$$
D^{2}(f)\left(x_{k}\right)=\frac{\sum_{j=1}^{n}\left(D^{1}(f)\left(x_{j}\right)-D^{1}(f)\left(x_{k}\right)\right)\left(x_{j}-x_{k}\right) p_{j}}{\sum_{j=1}^{n}\left(x_{j}-x_{k}\right)^{2} p_{j}}
$$

$$
\begin{aligned}
D^{2}(f)\left(x_{k}\right)= & \frac{\sum_{j=1}^{n}\left(f^{\prime \prime}\left(x_{k}\right)\left(x_{j}-x_{k}\right)^{2} p_{j}+\frac{f^{\prime \prime \prime}\left(\zeta_{j}\right)}{2}\left(x_{j}-x_{k}\right)^{3} p_{j}\right.}{\sum_{j=1}^{n}\left(x_{j}-x_{k}\right)^{2} p_{j}}+ \\
& \frac{\left.\sum_{j=1}^{n}\left\{\frac{\sum_{i=1}^{n} \frac{f^{\prime \prime}\left(\zeta_{i}\right)}{2}\left(x_{i}-x_{k}\right)^{3} p_{i}}{\sum_{i=1}^{n}\left(x_{i}-x_{k}\right)^{2} p_{i}}-\frac{\sum_{i=1}^{n} \frac{f^{\prime \prime}\left(\zeta_{i}^{\prime}\right)}{2}\left(x_{i}-x_{j}\right)^{3} p_{i}}{\sum_{i=1}^{n}\left(x_{i}-x_{j}\right)^{2} p_{i}}\right)\right\}\left(x_{j}-x_{k}\right) p_{j}}{\sum_{j=1}^{n}\left(x_{j}-x_{k}\right)^{2} p_{j}}
\end{aligned}
$$




$$
\begin{aligned}
& D^{2}(f)\left(x_{k}\right)-f^{\prime \prime}\left(x_{k}\right)= \frac{\sum_{j=1}^{n} \frac{f^{\prime \prime \prime}\left(\zeta_{j}\right)}{2}\left(x_{j}-x_{k}\right)^{3} p_{j}}{\sum_{j=1}^{n}\left(x_{j}-x_{k}\right)^{2} p_{j}} \\
&+\underbrace{\frac{\left.\sum_{j=1}^{n}\left\{\frac{\sum_{i=1}^{n} \frac{f^{\prime \prime}\left(\zeta_{j}\right)}{2}\left(x_{i}-x_{k}\right)^{3} p_{i}}{\sum_{i=1}^{n}\left(x_{i}-x_{k}\right)^{2} p_{i}}-\frac{\sum_{i=1}^{n} \frac{f^{\prime \prime}\left(\zeta_{j}^{\prime}\right)}{2}\left(x_{i}-x_{j}\right)^{3} p_{i}}{\sum_{i=1}^{n}\left(x_{i}-x_{j}\right)^{2} p_{i}}\right)\right\}\left(x_{j}-x_{k}\right) p_{j}}{\sum_{j=1}^{n}\left(x_{j}-x_{k}\right)^{2} p_{j}}}_{S_{2}} \\
& S_{2}=\frac{\left.\sum_{j=1}^{n}\left\{\frac{\sum_{i=1}^{n} \frac{f^{\prime \prime}\left(\zeta_{j}\right)}{2}\left(x_{i}-x_{k}\right)^{3} p_{i}}{\sum_{i=1}^{n}\left(x_{i}-x_{k}\right)^{2} p_{i}}-\frac{\sum_{i=1}^{n} \frac{f^{\prime \prime}\left(\zeta_{j}^{\prime}\right)}{2}\left(x_{i}-x_{j}\right)^{3} p_{i}}{\sum_{i=1}^{n}\left(x_{i}-x_{j}\right)^{2} p_{i}}\right)\right\}\left(x_{j}-x_{k}\right) p_{j}}{\sum_{j=1}^{n}\left(x_{j}-x_{k}\right)^{2} p_{j}}
\end{aligned}
$$

We note $h$ the discretization step. We call $\left(x_{i}-x_{k}\right)=\delta_{i k} h$ :

$$
S_{2}=\frac{\left.\sum_{j=1}^{n} \delta_{j k} h p_{j}\left\{\frac{\sum_{i=1}^{n} \frac{f^{\prime \prime}\left(\zeta_{j}\right)}{2} \delta_{i k}^{3} h^{3} p_{i}}{\sum_{i=1}^{n} \delta_{i k}^{2} h^{2} p_{i}}-\frac{\sum_{i=1}^{n} \frac{f^{\prime \prime}\left(\zeta_{j}^{\prime}\right)}{2} \delta_{i j}^{3} h^{3} p_{i}}{\sum_{i=1}^{n} \delta_{i j}^{2} h^{2} p_{i}}\right)\right\}}{\sum_{j=1}^{n} \delta_{j k}^{2} h^{2} p_{j}} .
$$

We lay $\Delta_{i k}=\frac{\delta_{i k}^{3} p_{i}}{\sum_{i=1}^{n} \delta_{i k}^{2} p_{i}}$

$$
S_{2}=\frac{\sum_{j=1}^{n} \delta_{j k} h p_{j}\left\{\sum_{i=1}^{n} f^{\prime \prime}\left(\zeta_{i}\right) \Delta_{i k}-f^{\prime \prime}\left(\zeta_{i}^{\prime}\right) \Delta_{i j}\right\}}{\sum_{j=1}^{n} \delta_{j k}^{2} h^{2} p_{j}}
$$

By taking the maximum:

$$
\begin{aligned}
S_{2} & \leq \max _{i, j \in I}\left\{\frac{\sum_{j=1}^{n} \frac{\delta_{j k}^{2} h^{2} p_{j}}{\delta_{j k} h}\left\{\sum_{i=1}^{n} f^{\prime \prime}\left(\zeta_{i}\right) \Delta_{i k}-f^{\prime \prime}\left(\zeta_{i}^{\prime}\right) \Delta_{i j}\right\}}{\sum_{j=1}^{n} \delta_{j k}^{2} h^{2} p_{j}}\right\} \\
& \leq \max _{i, j \in \mathcal{V}_{k}}\left\{\left|\frac{n}{\delta_{i k} h}\left(f^{\prime \prime}\left(\zeta_{i}\right) \Delta_{i k}-f^{\prime \prime}\left(\zeta_{i}^{\prime}\right) \Delta_{i j}\right)\right|\right\}
\end{aligned}
$$

We deduce the bounded error for second order derivative:

$$
\left|D^{2}(f)\left(x_{k}\right)-f^{\prime \prime}\left(x_{k}\right)\right| \leq \max _{j \in \mathcal{V}_{k}}\left\{\left|\frac{f^{\prime \prime \prime}\left(\zeta_{j}\right)}{2}\left(x_{j}-x_{k}\right)\right|\right\}+\max _{i, j \in \mathcal{V}_{k}}\left\{\left|\frac{n}{\delta_{i k} h} f^{\prime \prime}\left(\zeta_{i}\right) \Delta_{i k}-f^{\prime \prime}\left(\zeta_{i}^{\prime}\right) \Delta_{i j}\right|\right\}
$$

Corollary 15. Let $y_{i}=D^{1}(f)\left(x_{i}\right), x_{i} \in\left\{x_{1}, x_{2}, \ldots, x_{n}\right\}$ be the discretization of a function $y=f(x)$ with $\in \mathcal{C}^{3}$. Let $h$ be the discretization step. Then, when $h \rightarrow 0$, we have $\left|D^{1}(f)\left(x_{k}\right)-f^{\prime}\left(x_{k}\right)\right| \rightarrow 0$ and $\left|D^{2}(f)\left(x_{k}\right)-f^{\prime \prime}\left(x_{k}\right)\right| \rightarrow 0$

Proof. The study of the convergence of the bounded error could be found in 2 ] 


\subsection{Experimentation}

In this section, we propose examples to support the convergence of this estimator. We propose an estimation of the first derivative in Figure 1 We plot as well the estimation of the second derivative with a mask of length 50 and a discretization step of $\frac{1}{100}$ (Figure 3). Then we study the convergence of this estimation when the mask length is increased and the discretization step decreased. Whereas, in the first approximation there are some artifacts, we can see for a larger mask and a better digitalization step that we recover a good approximation (Figure[5] and Figure [6).

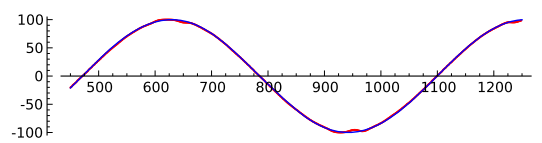

Fig. 1. First order derivative estimation for a mask of length 50 for $x \mapsto$ $\sin (x)$

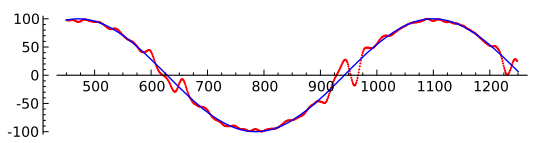

Fig. 3. Second order derivative estimation for a mask of length 50 for $x \mapsto$ $\sin (x)$

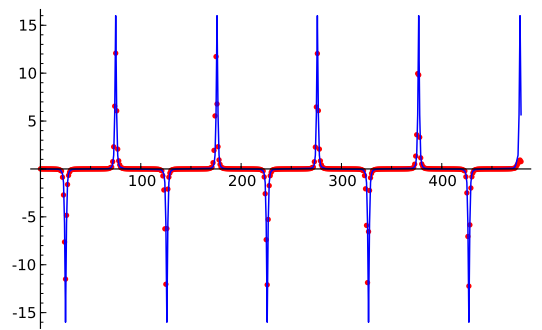

Fig. 5. Curvature estimation with a mask of length 300 and discrete step $h=\frac{1}{100}$

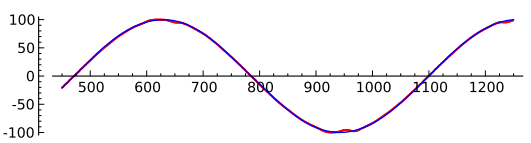

Fig. 2. First order derivative estimation for a mask of length 150 for $x \mapsto$ $\sin (x)$

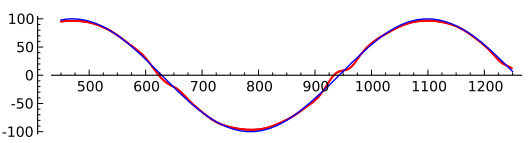

Fig. 4. Second order derivative estimation for a mask of length 600 for $x \mapsto \sin (x)$

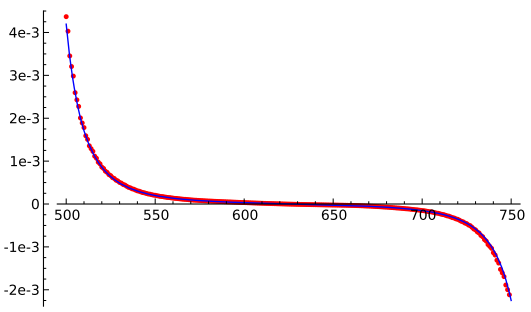

Fig. 6. Zoom on curvature estimation details Figure. 5

Fig. 7. Examples of curvature estimation on the function $x \mapsto \sin (x)$. Even for quite large discretization steps and small mask size, we have a reasonably good approximation of the curvature (Figure 5). But when considering smaller discretization steps, the estimation converges toward the exact values. 


\section{Conclusion}

In this article, we proposed a curvature estimator based on an adaptive kernel. Our approach is similar to a gaussian convolution on regular parts of the curve. First we have studied the link between the gaussian kernel and our approach. We proved that the gaussian process converges toward our adaptive estimator when the grid step converges toward zero and the mask length converges toward infinity. Then we used a least square method to bound the error between the estimation and the exact function with adaptive approach. This bounded error converges toward zero when the grid step converges toward zero and the length mask converges toward infinity. The issue of the length of the mask has not been studied in this paper and we have few information about the minimal length to have a satisfying estimation. In a forthcoming paper we will study the minimal length mask for a target bound of the error.

\section{References}

1. Alvarez, L., Lions, P.L., Morel, J.M.: Image selective smoothing and edge detection by nonlinear diffusion. ii. SIAM Journal on Numerical Analysis 29(3), 845-866 (1992)

2. An, Y., Shao, C., Wang, X., Li, Z.: Geometric properties estimation from discrete curves using discrete derivatives. Computers \& Graphics 35(4), 916-930 (2011)

3. Coeurjolly, D., Miguet, S., Tougne, L.: Discrete Curvature Based on Osculating Circle Estimation. In: Arcelli, C., Cordella, L.P., Sanniti di Baja, G. (eds.) IWVF 2001. LNCS, vol. 2059, pp. 303-312. Springer, Heidelberg (2001)

4. Coeurjolly, D., Sivignon, I., Tougne, L., Dupont, F. (eds.): DGCI 2008. LNCS, vol. 4992. Springer, Heidelberg (2008)

5. Esbelin, H.-A., Malgouyres, R.: Convergence of Binomial-Based Derivative Estimation for $C^{2}$ Noisy Discretized Curves. In: Brlek, S., Reutenauer, C., Provençal, X. (eds.) DGCI 2009. LNCS, vol. 5810, pp. 57-66. Springer, Heidelberg (2009)

6. Feschet, F., Tougne, L.: Optimal Time Computation of the Tangent of a Discrete Curve: Application to the Curvature. In: Bertrand, G., Couprie, M., Perroton, L. (eds.) DGCI 1999. LNCS, vol. 1568, pp. 31-40. Springer, Heidelberg (1999)

7. Fiorio, C., Mercat, C., Rieux, F.: Curvature Estimation for Discrete Curves Based on Auto-adaptive Masks of Convolution. In: Barneva, R.P., Brimkov, V.E., Hauptman, H.A., Natal Jorge, R.M., Tavares, J.M.R.S. (eds.) CompIMAGE 2010. LNCS, vol. 6026, pp. 47-59. Springer, Heidelberg (2010)

8. Fiorio, C., Mercat, C., Rieux, F.: Adaptive Discrete Laplace Operator. In: Bebis, G., Boyle, R., Parvin, B., Koracin, D., Wang, S., Kyungnam, K., Benes, B., Moreland, K., Borst, C., DiVerdi, S., Yi-Jen, C., Ming, J. (eds.) ISVC 2011, Part II. LNCS, vol. 6939, pp. 377-386. Springer, Heidelberg (2011)

9. Fourey, S., Malgouyres, R.: Normals and curvature estimation for digital surfaces based on convolutions. In: Coeurjolly, et al. (eds.) [4], pp. 287-298

10. Lachaud, J.-O., Vialard, A., de Vieilleville, F.: Fast, accurate and convergent tangent estimation on digital contours. Image Vision Comput. 25(10), 1572-1587 (2007)

11. Lindenbaum, M., Fischer, M., Bruckstein, A.M.: On gabor's contribution to image enhancement. Pattern Recognition 27(1), 1-8 (1994) 
12. Malgouyres, R., Brunet, F., Fourey, S.: Binomial convolutions and derivatives estimation from noisy discretizations. In: Coeurjolly, et al. (eds.) [4], pp. 370-379

13. Perona, P., Malik, J.: Scale-space and edge detection using anisotropic diffusion. IEEE Trans. Pattern Anal. Mach. Intell. 12(7), 629-639 (1990)

14. Provot, L., Gérard, Y.: Estimation of the Derivatives of a Digital Function with a Convergent Bounded Error. In: Debled-Rennesson, I., Domenjoud, E., Kerautret, B., Even, P. (eds.) DGCI 2011. LNCS, vol. 6607, pp. 284-295. Springer, Heidelberg (2011)

15. Roussillon, T., Lachaud, J.-O.: Accurate Curvature Estimation along Digital Contours with Maximal Digital Circular Arcs. In: Aggarwal, J.K., Barneva, R.P., Brimkov, V.E., Koroutchev, K.N., Korutcheva, E.R. (eds.) IWCIA 2011. LNCS, vol. 6636, pp. 43-55. Springer, Heidelberg (2011)

16. Roussillon, T., Sivignon, I., Tougne, L.: Measure of circularity for parts of digital boundaries and its fast computation. Pattern Recognition 43(1), 37-46 (2010)

17. Weickert, J.: Anisotropic diffusion in image processing. Kaiserslautern (1996) 